\title{
Critical emancipatory reflection on establishing an equal, trusting relationship among surgery participants in clinical practice in China
}

Discussion

\author{
Yan-Ling Lai* \\ The Department of Anesthesiology \& Operating Theatre, Sun Yat-sen University Cancer Center, State Key Laboratory of Oncology in South China, \\ Collaborative Innovation Center for Cancer Medicine, Guangzhou, Guangdong 510060, China
}

Received: 21 October 2018; Accepted: 22 December 2018; Published: 20 March 2019

Abstract: Objective: Reflection is considered to be one of the important ways to learn from one's experience, and one should be encouraged to apply the skill of reflection in lifelong learning. The author used the critical emancipatory reflection theory to reflect on a practice issue, which was related to the relationship between doctors and nurses, and tries to become a lifelong reflective practitioner in clinical work. Methods: Smyth's reflective framework, which includes the steps describe, inform, confront, and reconstruct, will be used in this article to help the author to understand the process of reflection and improve the skill of reflection. Utilizing Smyth's reflective framework to reflect on an issue in practice allows the author to break the routine way of thinking and learn from experience, as well as providing a higher quality of service for patients.

Results: The theory of emancipatory reflection along with the critical reflection theory will be used to determine the beliefs and values that rule the author's action and derive how these are distinct from what the author is supposed to achieve. Besides, critical emancipatory reflection theory will be used to discover the dominant power structures in clinical practice; symbolic interaction and hegemony will be utilized to discover the factors that prevent the author from achieving the desired goals; socialization theory will be applied to facilitate the author in improving the professional identity.

Conclusions: Using the critical emancipatory reflection on the issue in practice helps the author to find out the constraints in practice, bridge the espoused value and enacted value, and thereafter undertake transformative changes in practice. Eventually, the author can improve the skill of critical emancipatory reflection and become a lifelong reflective practitioner, and the quality of clinical practice can be improved as a result.

Keywords: critical emancipatory reflection $\bullet$ Smyth's framework • reflective practice $\bullet$ working relationship $\bullet$ patient-centered service

(c) Shanxi Medical Periodical Press.

\section{Introduction}

Reflection, which is considered to be one of the important ways to learn from experience, ${ }^{1}$ first introduced as a concept in 1980s, has developed rapidly in the past few decades, ${ }^{2}$ and its benefits for professional practice are being increasingly recognized. ${ }^{3}$ In some specializations, the skill of reflection is considered to be a defining feature of competence. ${ }^{2}$ Reflection inspires practitioners to focus on practice rather than on theory or technology; critical reflection frees the practitioners from ideology and fosters their understanding of clinical practice, encourages

How to cite this article: Lai YL. Critical emancipatory reflection on establishing an equal, trusting relationship among surgery participants in clinical practice in China. Front Nurs. 2019; 1: xx-xx. 
the practitioners to learn something new from experience and then find a better approach to practice, ensuring that a perspective of caring accompanies the whole process of practice; ${ }^{3}$ so, practitioners should be encouraged to apply the skill of reflection in lifelong learning. ${ }^{4,5}$ In order to master the process and skills of reflection, the author will reflect on the issue of establishing an equal, trusting relationship among surgery participants in practice and thereby obtain a better approach for clinical practice, as a result providing patient-centered services for patients and improving the quality of service.

\section{Methods}

A number of reflective models have been developed, making it easier for practitioners to reflect on practice, such as Gibbs' Reflective Cycle, Kolb's Experiential Learning Cycle, John's Model of Structured Reflection, and Smyth's reflective framework. In this article, Smyth's reflective framework with four stages will be used to guide the author's reflection process. Dewey ${ }^{6}$ defined reflection as a specialized form of thinking, which has a directly relationship with the situations that a person has experienced. For that reason, the author will use the first person "I" instead of the third person in this article, which would highly match the logic of the reflection process.

\section{Results}

\subsection{Describe}

After I graduated from university, I have been working in the Sun Yat-sen University Cancer Center for more than 5 years. I am an operating nurse; I work together with an anesthetist to assist the surgeon to implement and complete the surgery, and our common goal is to complete the operation safely and effectively. The psychological characteristics of patients who undergo surgery are quite different from those of others. Patients who are going to undergo surgery are anxious about surgery and feel panic. In addition, they are going to enter a completely unfamiliar environment without family and friends, and this exacerbates the patient's anxiety and the feelings of panic. ${ }^{7}$ Patients know little about the surgery, and this also makes them anxious and worried. As a nurse, I have the responsibility to reduce the patient's anxiety and help them eliminate their negative emotions. However, my work schedule is so busy that the time available for me to communicate with patients is limited. In practice, it is difficult for me to communicate with patients well or fully answer their questions. As an operating room nurse, confirmation of patient's information is an important way to ensure the safety of patients, so I will repeatedly confirm the patients' information with them. As a result, most of the communication with patients focuses on the confirmation of the information that I need, and the patients' questions and emotional needs tend to be ignored. As mentioned above, my work involves a close relationship with the anesthetist and surgeon; thus, it is not an independent process. I have tried to communicate to the anesthetist and surgeon regarding the need for about 10-15 minutes to communicate with my patients and answer patients' questions to meet their emotional needs. However, my opinion has not been accepted. The surgeon and anesthetist will only pay their attention after finishing the operation safely, and that too for as short a time as possible. They think that if even each patient needs a little time, the total working hours will increase a lot, and this will bring about an interruption into their work rhythm. Besides, they do not think that my spending time communicating with patients or answering patients' questions can effectively reduce the patients' negative emotions. In their opinion, their work is more valuable, and the value of my work is not recognized.

\subsection{Inform}

Reflection requires us to look at our own experience objectively. ${ }^{8}$ Reflection helps us to avoid taking our actions or behavior for grant. ${ }^{9}$ I try to objectively look at my behavior and understand what my behavior means. Similarly, critical reflection is also an approach to research our own experience and help us to have a better understanding of ourselves. ${ }^{10}$

It is the nurses' responsibility to deliver patient-centered care for patients. ${ }^{11}$ Taking into account the characteristics of surgical patients, they easily become anxious since they worry about surgery, and most of them feel panic. ${ }^{7}$ In addition, they are going to enter a completely unfamiliar environment without family and friends, which exacerbates the patients' anxiety and the feelings of panic ${ }^{7}$. To deliver patient-centered care, I should pay more attention to patients' emotional needs. However, the reality is that I lack engagement in patient-centered communication; in contrast, my communication with patients is a task-orientated communication. In addition, I do not answer patients' questions well enough, and this might increase the patients' negative emotions and make the patient feel that I lack compassion. Once, a patient said that medical staffs act like robots, making him feel that he was not respected. His words shocked me. As a nurse, being a patient-centered worker is very important, which directly relates to the quality of clinical practice $^{11}$. To obtain a better clinical outcome, I should take time to communicate with patients and help them to reduce their negative emotions when they need. 
This can greatly improve the patients' feelings, which is consistent with a nurse's values, such as empathy and compassion. ${ }^{12}$

In the operating room, the patient's safety is put at the first place, and all our works are based on the need to ensure patient safety. Therefore, repeated confirmation of patients' information is particularly important in our clinical practice, making the operative environment based on a culture that emphasizes the safety of patients. According to Fook and Askeland, ${ }^{13}$ culture is the sum total of beliefs and values shared by a group of people, and culture will affect its members' ideas and behaviors. Members in this group will take behavior that meets the cultural requirements for granted. In my case, influenced by the culture that emphasizes patients' safety, I value the safety of patients most and pay more attention to patients' safety first. As a result, I will focus on things that I think are more important, such as confirmation of patient's information, when time is limited. Furthermore, other medical staff put patients' safety at the first place, and the communication with anesthetists or surgeons also focuses on the confirmation of surgical information. In their opinion, the observation that patients feel nervous and panicked is very common, so we do not need to provide special solutions. I have tried to demonstrate my opinion that, in addition to patients' safety, the patients' emotional needs are also an important part of quality service. To achieve quality service, which is promoted by our hospital, we need to focus on patients' emotional needs as well as patients' safety. However, my opinion has been ignored.

The completion of each surgery requires the cooperation of nurses, anesthetists, and surgeons. Collaboration is considered to be one of the essential factors to guarantee a higher quality of health-care service. ${ }^{14,15}$ In a team such as ours, each one's work will directly affect the work of others; if I cannot complete my work effectively and on time, I will delay the process of the surgery and waste other persons' time. In this case, if I spend a lot of time to communicate with patients, it is possible that I will be complained about by the anesthetists and surgeons, and even be criticized by my leader. Anesthetists or surgeons do not accept my opinions, because the surgeon thinks that this team should be centered on them, so their opinions counts. As a result, I always feel upset and powerless in my clinical practice.

\subsection{Confront}

When informing the issue that I met in my daily work, it is obvious that there are a number of contradictions exist between my espoused value and enacted value, and this is also the case for belief. According to Schön, ${ }^{16}$ in many professionals, it is quite common that a gap exists between espoused and enacted beliefs. These contradictions prevent me from exhibiting a better professional performance. Emancipatory reflection requires me to deeply, systematically, and directly reflect on my experience to find out what is maintaining the problems that constrain my effective practice. ${ }^{17}$ Therefore, I need to find out the reasons behind my behavior, which makes me to take the behavior for granted. Fook and Gardner ${ }^{18}$ suggested that understanding what belief rules our behaviors and how this belief is different from our espoused belief, as well as learning from the findings, is the best way to help you make a change. To make a change, the critical reflection theory can be used to help us to implement this process, integrating and analyzing personal beliefs, the ways in which power is exercised, and the effects of dominant social thinking. ${ }^{19}$

In my case, the espoused values and beliefs comprise providing patient-centered health-care service, teamwork, and collaboration with respect and equality, which is related to the quality of clinical service. ${ }^{20}$ Providing patient-centered service is very important, which directly relates to the quality of clinical outcome. ${ }^{11}$ According to Shahriari, ${ }^{21}$ compassion is one of the key values that nurses should pay attention to. I would like to provide a better service for my patients, trying my best to achieve a patient-centered service. In order to achieve this goal, I need to show my compassion to my patients and pay more attention to patients' emotional needs. Collaboration is considered to have a very close relationship with a higher-quality health-care service. ${ }^{14}$ Furthermore, collaboration between different professions is based on mutual trust among team members and acknowledgment of professional knowledge. ${ }^{22}$ For members within a team, receiving respect from other members and obtaining equity are beneficial for all stakeholders, as well as for their patients.

Socialization basically is the process of learning throughout our life, and it fundamentally influences our beliefs and behaviors, ${ }^{23}$ which means internalizing the ideologies and norms of society. ${ }^{24}$ Socialization consists of teaching and learning, achieving both cultural and social continuity. ${ }^{24}$ After I have clarified the difference between my espoused faith and value on the one hand and the enacted faith and value on the other, socialization theory can be used to confront my espoused value and enacted value. Confucian culture is China's traditional cultural thought, which has deeply affected China for $>2,000$ years. Today, Confucian culture is still rooted in every Chinese mind, affecting our thoughts and behaviors. One of the characteristics of Confucian culture is patriarchy; as a result, paternalism is universally and deeply rooted within a lot of Chinese organizations. ${ }^{25} \mathrm{My}$ hospital is not an exception. According to Young et al., ${ }^{26}$ professional socialization involves gaining knowledge, 
skills, beliefs, and values to internalize and develop a professional identity. In my hospital, the vast majority of nurses are bachelor's degree holders, while almost all doctors have doctoral degrees. I usually lack confidence when I make my own suggestions. In addition, as the doctor's degree is generally higher than that of the nurses, the doctor's professional value has been recognized. On the contrary, the professional value and professional identity of nurses has long been neglected. ${ }^{27}$ This situation makes doctors feel that in this team, they are in the leading position, resulting in a doctor-dominated paternalism. As they think they are in charge of this team, they have the right to make decisions and judgments. According to Molleman et al., ${ }^{28}$ doctors usually think that collaboration will reduce their professional power; hence, they would not want to trust other team members. When I suggest that I need more time to focus on the patients' emotional needs and achieve patient-centered nursing, the doctors will not accept my opinion because they think that the effect of nursing on a patient's treatment is limited. It is the doctor's work that counts most. Similarly, affected by patriarchy, which is the characteristic of Confucian culture ${ }^{25}$, my grandfather and father have the power to decide things within our family. Often, if my opinions are different from theirs, they will not accept my opinion. As a result, I usually lack the courage to strive hard for my own opinion. This situation is very common in Chinese families. Chinese people generally are affected by the Confucian culture; the patient usually does not take the initiative to ask his/ her doctor for his/her own demands, because doctors have absolute authority over them. Moreover, when we are busy or lack patience, the patients are more afraid to express their feelings.

At the same time, economic factor is one of the important reasons that prevent me from achieving my espoused value and belief. In China, the health-care system is facing a shortage of nurses, causing a lot of problems. The number of nurses is small, and the demand for nurses is growing; hence, the attendant problem is that nurses have to work overtime. Working overtime will pose a threat to patients' safety and the quality of nursing, making the nurse overemphasize the importance of completion of their work over communicating with patients. ${ }^{29-31}$ Take myself, for instance, although I value the significance of patient-centered nursing, sometimes, it is hard for me to effectively and completely implement it. I have to work more than 10 hours every day. At the beginning, I work with passion and I try to do my work as perfectly as possible. However, during my overtime period, what I want most is to finish my work as quickly as I can. As a result, patients' emotional needs and communication needs tend to be ignored; furthermore, I am not willing to spend too much time and energy on either maintaining my professional identity or trying to establish an equal and trustful relationship with doctors.

\subsection{Reconstruct}

Through the stage of confronting, I have explored all the constraints that underpin my enacted values and beliefs in my clinical work. I realized that, in addition to myself, my work outcome was influenced by many other factors. According to Young et al. ${ }^{26}$ a professional identity can be developed and internalized by gaining knowledge and skill; consequently, a professional role can be acknowledged. If members of a team can trust each other and recognize each other's professional knowledge and skills, then multiprofessional teamwork and collaboration can be carried out, ensuring highquality service..$^{15,20,22}$ It is vitally important for me to learn constantly, enriching my professional knowledge and improving my professional skills. Only then will I have the confidence and ability to fulfill my professional role and change the doctor-dominated paternalism model. Furthermore, I will try to obtain the doctors' trust and communicate with them to establish a good relationship in our collaboration. This also requires me to have rich professional knowledge and proficiency in professional skills. It is suggested by McCormack et al. ${ }^{32}$ that lifelong learning practice can help me to achieve this goal, promoting the growth of our expertise and the improvement of professional skills. Moreover, it is essential to employ more nurses to ease my existing work pressure and liberate me from the overloaded working status. Then, I will have more time and energy to deliver higher-quality nursing for my patients.

In addition, after communicating with the doctor, I have tried to change my workflow. I made a suggestion to my leaders that we should do a good job in the preoperative visits, and this opinion has been accepted. One day before the patient's surgery, I will communicate with the patient in the ward; on the one hand, I can know more about the patient's situation in advance and I will have more time to answer their questions about the surgery. On the other hand, I can be a bit more familiar with patients in advance, which can help alleviate the anxiety of the patient. Meanwhile, I will encourage my patients to express their feelings and make requests whenever they need.

\section{Discussion}

By using the critical emancipatory reflection on the issue in my practice, I began to be aware of how my beliefs, values, feelings, and behaviors are influenced by external 
factors. Then, I started to explore the gap between my espoused value and my enacted value, as well as the relationship between these constraints and my work outcome through the stages of informing and confronting. After that, I realized that I should and have the ability to make a change rather than maintaining the status quo and doing nothing. First of all, I need to recognize and understand what beliefs and values I want to hold in my clinical practice. Patient-centered nursing is important and worthwhile for me to implement in my practice. Considering the characteristics of patients who are going to have surgery, I need to pay more attention to their emotional needs. After that, I need to equip myself with professional knowledge and professional skills, internalizing and developing my professional identity. ${ }^{26}$ For that reason, I studied in Ireland for the degree of Master of Science in Nursing in order to increase my professional knowledge and improve my professional skill, and as a result, to gain confidence to make my voice heard in my clinical work. A qualitative reflective research approach is an ideal way to bring about change in practice since awareness, new insight, and potential for improvement could be raised by research. ${ }^{33}$

\section{References}

1. Jasper M. Beginning Reflective Practice. United Kingdom: Nelson Thornes. 2003.

2. Loughran JJ. Effective reflective practice: in search of meaning in learning about teaching. $J$ Teach Educ. 2002;53:33-43.

3. Glaze JE. Reflection as a transforming process: student advanced nurse practitioners' experiences of developing reflective skills as part of an MSc programme. J Adv Nurs. 2001;34:639-647.

4. Forneris SG, Peden-McAlpine CJ. Contextual learning: a reflective learning intervention for nursing education. Int J Nurs Educ Scholarsh. 2006;3:1254.

5. Epp $\mathrm{S}$. The value of reflective journaling in undergraduate nursing education: a literature review. Int J Nurs Stud. 2008;45:1379-1388.

6. Dewey J. How We Think. New York: Heath. 1933.

7. Lee DS, Marsh L, Garcia-Altieri MA, Chiu LW, Awad SS. Active mental illnesses adversely affect surgical outcomes. Am Surg. 2016;82:1238-1243.

8. Somerville D, Keeling J. A practical approach to promote reflective practice within nursing. Nurs Times. 2004;100:42-45.

9. Thompson S, Thompson N. The Critically Reflective Practitioner. New York: Palgrave Macmillan. 2008.

10. Fook J. Reflective practice and critical reflection. In: Lishman J. ed. Handbook for Practice Learning
I should try to communicate with the doctor, obtain their trust, and strive to establish a cooperative relationship of equality and trust with them. I should express to the doctor my advice that we should pay more attention to patients' emotional needs and patients' safety and explain the reasons. ${ }^{8}$ I should also make a corresponding change in the workflow, visiting the patient before their surgery. By process optimization, I improve the quality of my work, provide a better service for patients, and gain the respect and trust of doctors.

\section{Conclusions}

Overall, the critical emancipatory reflection enabled me to learn from my work experience. I learned to think about the issue from a spectator's point of view and obtain a new perspective on this familiar problem. Consequently, I have the ability to make a transformative change and constantly improve the quality of my work.

\section{Conflicts of interest}

The author declares no conflicts of interest.

in Social Work and Social Care. London: Jessica Kingsley Publishers; 2007:440-454.

11. Health Service Executive. Guiding Framework for the Implementation of Nursing and Midwifery Quality Care-Metrics in the Health Service Executive Ireland. Ireland: Health Service Excusive. 2015.

12. de Vries J, Timmins F. Care erosion in hospitals: problems in reflective nursing practice and the role of cognitive dissonance. Nurse Educ Today. 2016;38:5-8.

13. Fook J, Askeland GA. Challenges of critical reflection: "nothing ventured, nothing gained". Soc Work Educ. 2007;26:520-533.

14. Al-Abri R. Managing change in healthcare. Oman Med J. 2007;22:9-10.

15. Zwarenstein M, Goldman J, Reeves S. Interprofessional collaboration: effects of practice-based interventions on professional practice and healthcare outcomes. Cochrane Database Syst Rev. 2009;3:CD000072.

16. Schön DA. Educating the Reflective Practitioner. San Francisco: Jossey-Bass. 1995.

17. Taylor B. Technical, practical, and emancipatory reflection for practicing holistically. $J$ Holist Nurs. 2004;22:73-84. 
18. Fook J, Gardner F. Practising Critical Reflection: A Resource Handbook. Berkshire: McGraw-Hill Education. 2007.

19 White S, Fook J, Gardner F. Critical Reflection in Health and Social Care. Berkshire: McGraw-Hill Education. 2006.

20. Coombs M, Ersser SJ. Medical hegemony in decision-making - a barrier to interdisciplinary working in intensive care? J Adv Nurs. 2004;46:245-252.

21. Shahriari M, Mohammadi E, Abbaszadeh A, Bahrami M. Nursing ethical values and definitions: a literature review. Iran J Nurs Midwifery Res. 2013;18:1-8.

22. Freeman M, Carolyn M, Nick R. The impact of individual philosophies of teamwork on multi-professional practice and the implications for education. $J$ Interprof Care. 2000;14:237-247.

23. Jakob C. Socialization. In: Brown K, ed. Encyclopedia of Language and Linguistics. North-Holland: Elsevier. 2006:462-466.

24. John JM. Sociology. 15th ed. Boston: Pearson. 2013:126.

25. Ma L, Tsui AS. Traditional Chinese philosophies and contemporary leadership. Leadership $Q$. 2015;26:13-24.
26. Young MN, Peng MW, Ahlstrom D, Bruton GD, Jiang Y. Corporate governance in emerging economies: a review of the principal-principal perspective. J Manage Stud. 2008;45:196-220.

27. Zelek B, Phillips SP. Gender and power: nurses and doctors in Canada. Int J Equity Health. 2003;2:1.

28. Molleman E, Broekhuis M, Stoffels R, Jaspers F. How health care complexity leads to cooperation and affects the autonomy of health care professionals. Health Care Anal. 2008;16:329-341.

29. Pelzang R. Time to learn: understanding patientcentered care. Br J Nurs. 2010;19:912-917.

30. Zolnierek $C$. The importance of knowing the patient. Issues Ment Health Nurs. 2011;32:392-393.

31. Bach S, Grant A. Communication and Interpersonal Skills in Nursing. 3rd ed. Nurse Educ Pract. 2015.

32. McCormack B, Dewing J, McCance T. Developing person-centered care: addressing contextual challenges through practice development. Online $J$ Issues Nurs. 2011;16:3.

33. Taylor B. Reflective Practice for Healthcare Professionals: A Practical Guide. 3rd ed. Berkshire: McGraw-Hill Education. 2010. 